\title{
El capital social local y sus determinantes: evidencia de Cataluña
}

\section{Sergi Sánchez Coll}

RESUMEN: Ante la creciente importancia del estudio del capital social como componente institucional y la escasez de estudios empíricos a menor escala y fuera de los Estados Unidos, este trabajo pretende identificar algunos de determinantes observables del capital social en los municipios y comarcas de Cataluña, así como su relación con los niveles de capital social y su distribución territorial. Esto se consigue mediante el uso de la participación electoral, la densidad asociativa y las donaciones de sangre como variables de capital social. Usando datos de corte transversal y de panel y mediante regresiones por mínimos cuadrados ordinarios y de efectos aleatorios respectivamente se encuentran efectos relevantes sobre el capital social del grado de movilidad residencial, la densidad de población y asociativa, el régimen de propiedad de la vivienda, la renta, el nivel educativo y la edad. En ciertos casos las relaciones se corresponden con las encontradas en la literatura existente. Además, extrayendo sus componentes principales se elabora un índice de capital social, con el cual se observa una distribución geográfica desigual del atributo, penalizando el Área Metropolitana de Barcelona y la costa.

PALABRAS CLAVE: Capital social, participación electoral, densidad asociativa, donaciones de sangre, movilidad residencial, municipios, comarcas, Cataluña, España.

CLAVES ECONLIT: D71, D72, P48, R12, Z13.

Cómo citar este artículo / How to cite this article: SÁNCHEZ-COLL, S. (2020): "El capital social local y sus determinantes: evidencia de Cataluña", CIRIEC-España, Revista de Economía Pública, Social y Cooperativa, 99, 203-232. DOI: 107203/CIRIEC-E.99.15706.

Correspondencia: Sergi Sánchez Coll, Universitat Pompeu Fabra - sergisanchezcoll@alumni.upf.edu.

ORCID: https://orcid.org/0000-0002-4012-2980. 


\section{EXPANDED ABSTRACT}

\section{Local social capital and its determinants: evi- dence from Catalonia}

\section{Purpose}

Since it gained popularity in the late 20th century, social capital has been widely studied for its key role in bonding societies while generating socially desirable outcomes, such as economic growth and development, political participation, innovation and entrepreneurship. Social capital levels and its determinants have generally been studied either individually or at a regional or national level, leaving the local level with little empirical evidence. In this regard, this paper aims to provide an overview of both the determinants and levels of social capital, as well as the patterns of their geographical distribution at the municipality and county level in the Catalonia region.

Specifically, three main hypotheses are considered in this study: 1. Local social capital in CataIonia has observable determinants. 2. The patterns described in similar empirical evidence for other territories regarding the determinants and distribution of local social capital are also present in the case of Catalonia. 3. Social capital is unequally distributed throughout the region.

\section{Approach}

The two main sources of conflict when studying social capital arise when dealing with its definition and its measurement, which has prevented the concept from gaining more importance. While some argue that it should not be called capital or that it is a community feature, the economic approach assumes that social capital is a feature of the individual. Regarding its measurement, survey data and proxies are the two main approaches followed in research.

This paper follows the individual approach of economics, which allows for econometric modelling, using data on three indirect measures of social capital: average turnout in recent local, regional and Spanish general elections, associational density and average blood donations. Catalan municipalities over 5,000 inhabitants and 41 counties were included in the data set, except the five biggest cities, which experience too much variation in neighbourhood characteristics to be considered single entities and are therefore excluded from the analysis.

Following the methodology of Becker (1965) and Rupasingha et al. (2006) regarding household allocation of time and social capital formation, the model assumes that the representative household of a municipality or county can invest in two items: composite consumption, which is a function of market goods and time devoted to their production, and social capital, a function of household social 
capital goods, the time allocated to their production, the household's own characteristics and the characteristics of other households. Investment in composite consumption implies the production of goods and activities, while social capital can produce observable social capital in the shape of associational density, election turnout or blood donations. The share of investment in each will depend on income restrictions, as well as on the amount of time available in each household and the prices of both market and social capital goods.

This framework allows for performing ordinary least squares regressions, and the annual series of the data on blood donations allows for a panel data analysis of seven consecutive years. Further, adding independent and control variables to the model allows for the observation of social capital determinants. Specifically, data on the prevalence of immigrants, elderly people and highly educated citizens in the municipality and county were included in the study. The income, population size, population density, percentage of home ownership, altitude of the municipality and a measure of geographical mobility, which was calculated using the proportion of people moving in and out of the city and county every year, respectively, were also considered. The sources of data were the Statistical Institute of Catalonia, the Government of Catalonia, the Catalan Blood and Tissue Bank and the Spanish National Statistics Institute.

\section{Findings}

The results show a negative relationship between the percentage of owned homes and social capital in the municipalities, although the theory suggests that owning a home increases the roots of the household in the area and so does social capital. However, other evidence has also encountered this counterintuitive result. Similarly, geographical mobility negatively affects election turnout and blood donations, especially in municipalities, in line with the evidence and the theories linking residential stability with the creation of stronger social networks. The percentage of immigrants is associated with smaller political participation, which is coherent with their limitations in voting rights. Other variables do not show any clear effect on social capital outcomes, such as income, which is predicted to have a positive coefficient.

Education has a limited and mostly positive effect, but it is far from the magnitude observed in the empirical evidence, which regards it as a key determinant of social capital. The proportion of elderly population seems to encourage the creation of associations and to discourage both blood donations, which in Catalonia are limited to people under 70, and turnout, which is counterintuitive since voting rates are traditionally high among older adults. The density of associations is clearly linked to higher turnout and blood donations. Altitude positively affects this density, so municipalities in mountainous areas tend to have more associations, while greater population density is related to less associational density and blood donations.

In addition, an index to summarise the levels of social capital was created by performing a principal component analysis using the three social capital proxies. The highest levels were found to be located in inland municipalities under 30,000 inhabitants, with few exceptions. Eight of the nine municipalities 
with the lowest values belong to the Barcelona province, with five in its metropolitan area, validating the idea that commuter cities have low levels of social capital. Coastal cities are also affected by this phenomenon, perhaps due to the seasonal character of housing. Regarding the highest concentrations of social capital, they were mostly found in municipalities located in the provinces of Girona and Lleida, in eastern and western Catalonia, which are sparsely populated.

The distribution of social capital in counties shows high levels in western counties and in the western part of the Girona province, while counties with access to the sea are generally poor in social capital. The main common characteristic of those areas is the predominance of sparsely populated towns with a few hundred inhabitants, since among the five counties with the highest values only the city of Ripoll contains more than 5,000 inhabitants and is therefore included in the study of the municipalities.

\section{Limitations}

The results of this study were subject to a number of limitations. The main issue has been the general lack of statistical data for municipalities under 5,000 inhabitants and the limited availability of statistics at the municipality and county level. This impedes the complete visualisation of the data, as well as the identification of causal impacts and the influence of age groups, family sizes, urban distributions, years of schooling and spending in health care and education on the outcomes. The suspected role that income inequality plays in social capital could not be checked due to the lack of recent data, while other data sources are only updated every decade.

Further research could analyse the production process of local social capital in Catalonia, its relationship with the life cycle and the characteristics of areas with high levels of social capital that could be useful for policy purposes. The use of other measuring scales for big cities, such as neighbourhoods, would be highly valuable.

The findings could gain precision when being able to use instrumental variables to find the causal impact, as well as when using the proportion of blood donations achieved in each campaign as a better measure of the intensity of blood donations. In addition, taking into account the role of geography and capital cities in each county would be highly relevant.

\section{Practical Conclusions and Original Value}

By studying three variables associated with social capital some of its main determinants have been identified in both municipalities and counties. Different outcomes in the county analysis were also observed in some cases. Some patterns go in line with the empirical evidence, so Catalonia is not an exception, but some differ, leaving room for future confirmation of those results.

A social capital index is useful to compare both municipalities and counties, despite the fact that most municipalities are too small to be in the study, showing a pattern that seems to harm coastal 
areas and the Barcelona metropolitan area and to benefit counties with a high prevalence of sparsely populated small towns.

This paper does not solve the issue of indirect measurement of social capital, nor the controversy surrounding the concept, but it might shed light on the local characteristics of social capital in Catalonia and its future research and policy implications. This contribution tries to explain those local differences with an unclear origin which promote social cohesion, with valuable insights for local public policy in terms of promoting and sustaining social capital.

KEYWORDS: Social capital, voter turnout, association density, blood donations, residential mobility, municipalities, counties, Catalonia, Spain.

\section{Introducción}

La primera mención conocida del capital social se remonta a hace más de cien años en el campo de la pedagogía (Hanifan, 1916), pero el concepto se popularizó sobretodo durante los años ochenta dentro de la sociología, y a partir de Putnam (Putnam, 1993) hizo el salto a otras ciencias sociales como la economía, donde adquirió una importancia creciente en el campo institucional y del desarrollo de las comunidades. En cuanto a su definición no hay consensos establecidos, y difieren ampliamente según la disciplina (Adler \& Kwon, 2002; Guiso, Sapienza, \& Zingales, 2011).

Dentro de la rama que considera al capital social como las relaciones que un actor mantiene con actores externos destaca la obra de Pierre Bourdieu, que lo define como «la suma de recursos corrientes o virtuales que se acumulan en un individuo o grupo a través de la posesión de una red durable de relaciones mutuas y reconocimiento, más o menos institucionalizadas" (Bourdieu, 1986: 249). Según Bourdieu, el capital social es una propiedad individual y hace una interpretación negativa del uso que se le puede dar, ya que considera que está ligado al estrato social al cual se pertenece y a las clases bienestantes. Muestra al capital social como una herramienta que tienen las élites para asegurarse de que la gente inadecuada no pueda acceder a sus círculos excluyentes (Bourdieu, 1986). También relaciona el concepto con la economía pues, bajo ciertas condiciones, se puede transformar en capital económico y puede ser institucionalizado en forma de título nobiliario.

Dentro del sector de autores que se centran en el capital social como la estructura de relaciones entre actores dentro de una comunidad destacan James Coleman y Robert Putnam. El primero considera que el capital social se define por su función, y que consiste en un conjunto de entidades que tienen en común el hecho de disponer de algún tipo de estructura social y el hecho de facilitar ciertas 
acciones a los individuos que están dentro de esta estructura (Coleman, 1988: S98). A diferencia de Bourdieu, Coleman considera a toda la sociedad como capaz de acumular capital social, también las personas desfavorecidas. Para Putnam, el capital social se entiende como «las características de una organización social, tales como redes, normas y confianza social que facilitan la coordinación y la cooperación para un beneficio mutuo» (Putnam, 1993: 167).

Por su parte, Nan Lin propone una teoría del capital social basada en la teoría de redes. Estas redes no necesariamente deben ser densas, cerradas o restrictivas, ya que el autor distingue entre acciones expresivas, donde el actor pretende mantener los recursos de capital colectivo que ya posee y por lo tanto le favorecería tener redes cerradas y densas, y acciones instrumentales, en las cuales se pretenden obtener unos mayores retornos económicos, políticos y sociales, y por lo tanto el actor puede beneficiarse de los puentes que unas redes sociales más abiertas y dispersas pueden ofrecer (Lin, 1999, 2005).

La importancia del capital social yace en las externalidades mayoritariamente positivas que puede producir. Estas incluyen la salud y menor mortalidad (Sundquist et al., 2014), el rendimiento económico (Peiró Palomino \& Tortosa-Ausina, 2012; Rupasingha, Goetz, \& Freshwater, 2000) y el desarroIlo, entendido como innovación y emprendimiento (Malecki, 2012). Asimismo, se relaciona al capital social con las diversas políticas de Responsabilidad Social Corporativa, así como con una reducción de la conflictividad entre empresa y sociedad (De Nieves Nieto \& Briones Peñalber, 2009). Se suele poner de ejemplo el caso de las Organizaciones No Lucrativas (ONL) como generadoras de capital social cuando son socialmente eficientes y bajo ciertas condiciones (Bellostas Pérez-Grueso, Marcuello Servós \& Moneva Abadía, 2007).

A pesar de los mencionados enfoques para su definición, diferentes autores son escépticos con el capital social, sea por la poca claridad del concepto, que se podría definir mejor con otras palabras como afiliación, familia, sociabilidad o confianza (Fischer, 2001), o bien por el uso inadecuado del término "capital" (Arrow, 2000; Fischer, 2001; Solow, 2000), y no hay consenso sobre qué papel le corresponde al sector público. Algunos académicos defienden que el Estado es necesario para promover el capital social mediante incentivos institucionales (Kenworthy, 1997). Otros, que es perjudicial, poniendo el ejemplo de las sociedades comunistas como destructoras del capital social previamente presente en las mismas (Nichols, 1996), si bien ciertas zonas excomunistas destacan por sus elevados niveles (Petro, 2001).

Engbers et al. proponen cinco grandes tipos de capital social: confianza social, membresía formal y participación, altruismo y participación política, interacción informal y reglas compartidas y homogeneidad, con una serie de variables representativas de los mismos en el caso de los Estados Unidos (Engbers, Thompson, \& Slaper, 2017). En el presente trabajo se utilizan variables correspondientes a todos los tipos excepto la confianza social. Además, al capital social se le pueden atribuir diferentes niveles de intensidad a nivel teórico, con diferentes efectos según la cantidad de capital social que se 
posee, afectando, entre otros, a los valores, las funciones de utilidad y, en teoría de juegos, a los tipos de juegos que tienen lugar y las estrategias a seguir en los mismos (Uphoff, 2000).

El segundo problema que presenta el capital social después del de la definición es el de su medición. Según Coleman, hay dos visiones opuestas según la disciplina académica que se considere (Coleman, 1988). Por un lado, la sociología suele considerar a los actores como sujetos a normas y obligaciones sociales, y por lo tanto al capital social como un atributo comunitario, aunque al actor no se le reconoce ningún tipo de impulso de acción propio. En cambio, la economía tiende a ver a individuos interesados en ellos mismos y en el cumplimiento de sus objetivos personales, siguiendo el principio neoclásico de maximización de la utilidad y obviando el entorno. Dentro de esta perspectiva destacan el modelo de Glaeser et al. y el de Fernández de Guevara et al. (Glaeser, Laibson, \& Sacerdote, 2002; Montesinos Santalucía, Pérez García, Serrano Martínez, \& Fernández de Guevara, 2005).

En referencia al tipo de datos, existen dos grandes tipos de aproximaciones a la medición del capital social: la primera usa datos de encuesta sobre el grado de confianza de la población, a menudo preguntando sobre confianza, frecuencia de los contactos vecinales, disponibilidad de redes sociales a quien poder consultar problemas, o participación individual en asociaciones. Como señalan Glaeser et al., sin embargo, esta medida subjetiva del capital social presenta algunos problemas, pues las preguntas son vagas, abstractas y difíciles de interpretar, y las respuestas están más relacionadas con los niveles de fiabilidad que con los de confianza (Glaeser, Scheinkman, \& Soutter, 1999).

La segunda aproximación a la medición del capital social busca variables cuantificables que lo expliquen, como pueden ser la densidad asociativa, las donaciones de sangre, la participación electoral o los lectores de periódicos. Este es el método que empezaron a desarrollar Putnam et al. en 1983 (Putnam, Leonardi, Nanetti, \& Pavoncello, 1983). La ventaja es que las medidas son mucho más fiables, pues son objetivas y se refieren a toda la población, pero no está claro que sean consecuencias exclusivas del capital social, y como mucho solo explican una parte. Para este análisis usaremos esta segunda aproximación.

Los determinantes del capital social local se han estudiado poco. Los pioneros en este tipo de estudios fueron Rupasingha et al., que estudiaron tanto los determinantes como los niveles de capital social en los condados de los Estados Unidos (Rupasingha, Goetz, \& Freshwater, 2006). Dada la escasez de evidencias posteriores a nivel regional y fuera de los Estados Unidos seguiremos su trabajo y modelo, aplicándolo a los municipios y comarcas de Cataluña. La Figura 1 muestra el diagrama explicativo del modelo seguido, a partir del de Becker y la posterior adaptación de Rupasingha et al., fundamentado en una perspectiva de inversión individual en consumo compuesto y capital social, de los cuales se pretende obtener utilidad (Becker, 1965; Rupasingha et al., 2006). Es relevante mencionar la mezcla que el hogar representativo hace entre producción y consumo, pues se asume que realiza ambas tareas al producir bienes que necesitan otros bienes para satisfacer su utilidad (por ejemplo, la preparación de una comida necesita diferentes ingredientes para llevarse a cabo). 


\section{Figura 1. Modelo de producción de capital social, basado en los modelos de Becker y Rupasingha et al.}

(Becker, 1965; Rupasingha et al., 2006)

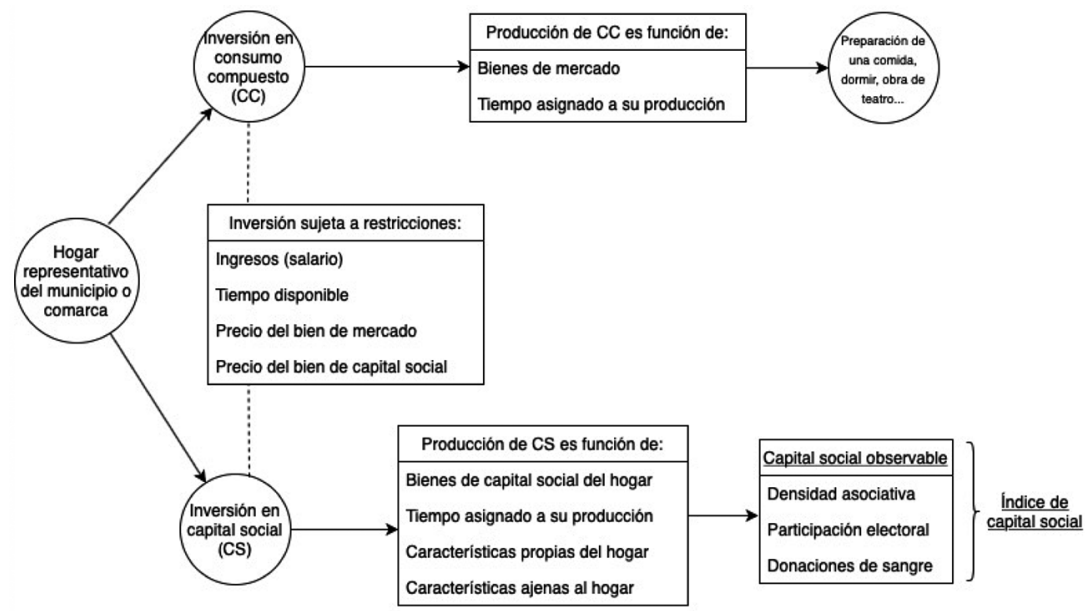

El hecho de estudiar las donaciones de sangre como sinónimo de capital social se puede justificar en relaciones ya establecidas en la literatura (Bekkers \& Veldhuizen, 2008; Buonanno, Montolio, \& Vanin, 2009; Smith, Matthews \& Fiddler, 2011). Los incentivos no monetarios son clave a la hora de mantener tanto los niveles de donación de sangre, en la línea de Titmuss (Mellström \& Johannesson, 2008), como la calidad sanitaria de las mismas (Poel, Seifried, \& Schaasberg, 2002).

En cuanto a la densidad asociativa, ha sido ampliamente tratada como proxy del capital social (Buonanno et al., 2009; Maloney \& Rossteutscher, 2007; Putnam, 1993; Rupasingha et al., 2006). A nivel municipal en Cataluña ha sido estudiada recientemente, aunque centrándose en el papel de los bienes comunales como determinantes históricos del capital social actual (Montolio \& Tur-Prats, 2018).

Por otro lado, Montero et al. han analizado la relación entre confianza social, confianza política, confianza en las instituciones y satisfacción con la democracia en Cataluña (Montero, Zmerli, \& Newton, 2008). Concluyen que la confianza social forma parte de un círculo virtuoso que se asocia con la participación comunitaria, la pertenencia voluntaria a asociaciones, todo esto a la vez asociado a una mayor implicación cívica y participación política democrática. La relación entre capital social y comportamiento electoral es encontrada por Putnam de modo indirecto para las regiones italianas, pues hay factores históricos que contaminan la participación electoral, pero usa la participación en referéndums para la elaboración de su índice de comunidad cívica. (Putnam, 1993). Posteriormente 
ha habido numerosos usos de la concurrencia a las urnas tanto en referéndums como en elecciones (Buonanno et al., 2009; Dipasquale \& Glaeser, 1999; McCabe, 2013; Rupasingha et al., 2006).

El objetivo del presente estudio empírico es doble. En primer lugar, se pretende identificar los factores que determinan la creación de capital social en los municipios catalanes mayores de 5.000 habitantes y en las comarcas, a partir de una definición de capital social que incluye tres variables generalmente representativas de sus niveles como son la densidad asociativa, las donaciones de sangre y la participación electoral. Mediante modelos econométricos de mínimos cuadrados ordinarios para los datos transversales y de efectos aleatorios para los datos de panel, así como una base de datos de variables independientes y de control, se pretende encontrar asociaciones entre variables que permitan explicar los factores que favorecen la creación de capital social.

En segundo lugar, se pretende conocer los respectivos niveles de capital social con la creación de un índice sintético de capital social mediante un análisis de componentes principales de las tres variables consideradas como parte de este. Además, con la representación geográfica del capital social se aspira a dar una visión que permita encontrar patrones que puedan explicar las importantes diferencias en las dotaciones que se producen.

Las hipótesis son las siguientes: 1. El capital social local tiene determinantes observables. 2. El capital social local sigue en Cataluña los mismos patrones que los descritos en estudios para otros territorios. 3. El capital social local está distribuido desigualmente por el territorio. No hay razones para pensar que el caso de Cataluña tenga que ser diferente en cuanto a determinantes, y se espera que el capital social tenga valores bajos en el Área Metropolitana de Barcelona y altos en municipios interiores con poca movilidad.

El trabajo continúa de la siguiente forma: en la sección 2 se presentan los datos con los cuales se ha realizado el estudio, sus características principales, los métodos usados en el análisis para la identificación de los factores que favorecen unos mayores niveles de capital social y los fundamentos teóricos del análisis. La sección 3 presenta los resultados del estudio para las tres variables estudiadas como parte del capital social, así como el índice de capital social y su distribución geográfica. A continuación, la sección 4 abre una discusión sobre las implicaciones de los resultados obtenidos y las dificultades encontradas, comparándolos con la evidencia existente. Finalmente, la conclusión cierra la investigación. 


\section{Datos y método}

El estudio se ha llevado a cabo a partir de la creación de una base de datos de todos los municipios catalanes mayores de 5.000 habitantes, en el periodo estudiado según los datos disponibles, y de 41 comarcas, para las variables detalladas en los siguientes párrafos. Los motivos para limitar la investigación a los mayores de 5.000 habitantes son dos: en primer lugar, para muchos datos esta dimensión suele ser el límite a partir del cual se realiza una estadística en un municipio, de modo que los datos solamente están disponibles para los de este tamaño y superiores. En segundo lugar, en los municipios más pequeños los determinantes del capital social pueden estar contaminados por el hecho de que su producción puede ser facilitada por los mayores vínculos sociales que la dimensión reducida permite crear.

Se han omitido los municipios de nueva creación (Medinyà y La Canonja), Matadepera, que no disponía de datos de donaciones de sangre y, aunque se mantienen en los mapas descriptivos, las cinco mayores ciudades de Cataluña en número de habitantes, (Badalona, Barcelona, l'Hospitalet de Llobregat, Sabadell y Terrassa), pues es relevante la influencia que tienen en los datos de población, con una desviación típica muy superior a la media, y probablemente sería más informativo estudiarlos a menor escala, a nivel de barrio o distrito. En el caso de las comarcas se ha omitido el Moianès, por ser de reciente creación justo en la mitad del periodo estudiado.

Se ha dispuesto de una base de datos del número de donaciones de sangre municipales y comarcales realizadas en las últimas siete campañas por el Banco de Sangre y Tejidos de Cataluña (2012 a 2018). La densidad asociativa se ha medido a partir de la lista del número de asociaciones y federaciones registradas en la Guía de Entidades del Departamento de Justicia de la Generalitat de Catalunya en cada municipio o comarca, consultada en abril de 2019. Finalmente, los datos de participación electoral provienen del Instituto de Estadística de Cataluña (Idescat). En cuanto a las variables independientes y de control, los datos del régimen de propiedad de la vivienda, censo, renta familiar disponible media, densidad de población, altitud, nivel de instrucción de la población, porcentaje de población extranjera y número de migraciones internas también provienen de Idescat, mientras que los datos municipales y comarcales de población mayor de 65 años provienen de la Estadística del Padrón Continuo del Instituto Nacional de Estadística. A continuación, se presenta una definición de los datos estudiados. 
- partim: Media de la participación en porcentaje en las elecciones locales (2011 y 2015), autonómicas (2010, 2012, 2015 y 2017) y generales (2011, 2015 y 2016). El principal argumento para utilizar una media de las elecciones en los últimos ocho años es intentar aislar el componente del interés político que ciertas candidaturas o elecciones pudieran suscitar, pues puede afectar significativamente los niveles de participación.

- asoc: Número de asociaciones y federaciones registradas en el municipio o comarca por 1000 habitantes, abril de 2019.

- dm: Media de los índices de donación de sangre (donaciones por 1000 habitantes) en cada municipio y comarca, 2012-2018.

- eduter: Porcentaje de habitantes con educación terciaria (Ciclo Formativo de Grado Superior, diplomatura, grado, licenciatura y doctorado) completada, 2011.

- rentam: Media de la Renta Familiar Disponible Bruta, 2012-2018. Para los años 2017 y 2018 se ha extrapolado a partir de la tasa de crecimiento de los años anteriores.

- poblacionm: Media del censo anual de cada municipio o comarca expresado en número de habitantes, 2012-2018.

- logdpm: Logaritmo de la media de la densidad de población en habitantes por kilómetro cuadrado, 2012-2018.

- inm: Media del porcentaje de población extranjera, 2012-2018.

- movilidadm: Media de la medida de movilidad, 2012-2018. Esta medida se ha confeccionado sumando el número de inmigraciones y emigraciones internas recibidas por el municipio 0 la comarca en un determinado año, dividiendo el resultado por el censo correspondiente y expresándolo en porcentaje.

- maym: Media del porcentaje de habitantes mayores de 65 años, 2012-2018.

- propiedad: Porcentaje de viviendas en régimen de propiedad, 2011.

- alquiler: Porcentaje de viviendas en régimen de alquiler, 2011.

- altitud: Altitud del municipio en metros sobre el nivel del mar (msnm).

Las tablas 1 y 2 muestran las estadísticas descriptivas de las variables para los municipios y las comarcas. La desviación típica de la variable población media es superior a la media, debido al impacto de las ciudades más habitadas, pero menor que si se incluyeran los cinco municipios más poblados. Con la altitud también se observa una desviación típica superior a la media debido a los $1.202 \mathrm{msnm}$ en los que se encuentra situado el municipio de Puigcerdà, pues la mayoría de los municipios estudiados se sitúan por debajo de los $300 \mathrm{msnm}$, pero la influencia en los resultados es todavía menor. Asimismo, en los datos se aprecia una elevada variabilidad geográfica en los niveles de educación terciaria, densidad asociativa y régimen de tenencia de la vivienda, con distribución aproximadamente normal, así como en la inmigración y las donaciones de sangre, con concentración de datos en los valores bajos. También destaca la poca variación en la participación electoral de los distintos municipios y comarcas. 
Tabla 1. Estadísticas descriptivas de los datos municipales

\begin{tabular}{|l|r|r|r|r|r|}
\hline Variable & Obs & Mean & Std.Dev. & Min & Max \\
\hline partim & 200 & 66.98 & 3.15 & 58.98 & 73.91 \\
\hline asoc & 200 & 9.67 & 2.78 & 4.44 & 19.35 \\
\hline $\mathrm{dm}$ & 200 & 29.71 & 16.36 & 6.48 & 89.85 \\
\hline eduter & 200 & 22.67 & 6.69 & 10.25 & 48.05 \\
\hline rentam & 200 & 15.46 & 2.47 & 9.98 & 24.26 \\
\hline poblacionm & 200 & 21110.49 & 24102.35 & 4863.43 & 138669.7 \\
\hline logdpm & 200 & 6.51 & 1.3 & 3.01 & 9.74 \\
\hline inm & 200 & 12.86 & 8.03 & 2.2 & 49.74 \\
\hline movilidadm & 200 & 8.71 & 1.91 & 4.88 & 15.92 \\
\hline maym & 200 & 16.34 & 2.81 & 9.01 & 25.95 \\
\hline propiedad & 199 & 71.74 & 8.87 & 34.3 & 95.15 \\
\hline alquiler & 197 & 16.82 & 6.79 & 3.85 & 51.22 \\
\hline altitud & 200 & 178.34 & 191.39 & 2 & 1202 \\
\hline
\end{tabular}

Tabla 2. Estadísticas descriptivas de los datos comarcales

\begin{tabular}{|l|r|r|r|r|r|}
\hline Variable & Obs & Mean & Std.Dev. & Min & Max \\
\hline partim & 41 & 68.40 & 2.65 & 63.94 & 76.42 \\
\hline asoc & 41 & 14.13 & 5.41 & 6.65 & 34.65 \\
\hline $\mathrm{dm}$ & 41 & 38.54 & 13.11 & 15.41 & 72.19 \\
\hline eduter & 41 & 23.29 & 4.28 & 13.49 & 33.53 \\
\hline rentam & 41 & 15.07 & 1.37 & 12.1 & 18.98 \\
\hline poblacionm & 41 & 184076.9 & 382389.1 & 3936.714 & 2240368 \\
\hline logdpm & 41 & 4.55 & 1.68 & 1.64 & 9.64 \\
\hline inm & 41 & 14.45 & 4.24 & 7.86 & 25.83 \\
\hline movilidadm & 41 & 8.37 & 1.73 & 4.57 & 12.99 \\
\hline maym & 41 & 19.16 & 2.81 & 9.01 & 25.95 \\
\hline propiedad & 41 & 74.32 & 3.38 & 13.01 & 28.45 \\
\hline alquiler & 41 & 18.03 & 5.43 & 7.66 & 31.87 \\
\hline
\end{tabular}


Las figuras 2 a 4 muestran la distribución geográfica de los diferentes valores de las tres variables estudiadas, usando la herramienta Instamaps del Instituto Cartográfico y Geológico de Cataluña'. Concretamente, la Figura 2 muestra como la participación electoral tiene una variabilidad pequeña con respecto a las distintas observaciones y en el horizonte temporal. No se observa ningún patrón claro de voto en los municipios estudiados, si bien la participación es baja en la costa y en algunos municipios de la provincia occidental de Lleida, y los valores elevados tienden a estar concentrados en la provincia de Barcelona. A nivel comarcal se aprecia una baja participación en la costa, con unos niveles elevados en la Terra Alta y el Priorat, en el suroeste, ambas comarcas formadas exclusivamente por municipios menores de 5.000 habitantes, de modo que no aparecen en el análisis municipal.

\section{Figura 2. Participación electoral media municipal y comar- cal, 2010-2017 (en \%)}

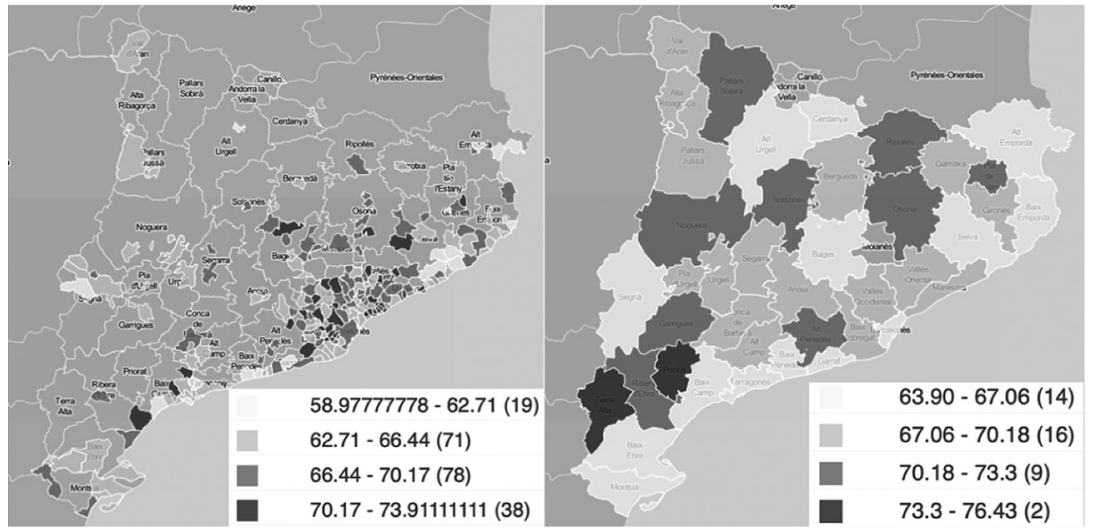

Figura 3. Densidad asociativa municipal y comarcal, 2019 (en asociaciones por 1.000 habitantes)

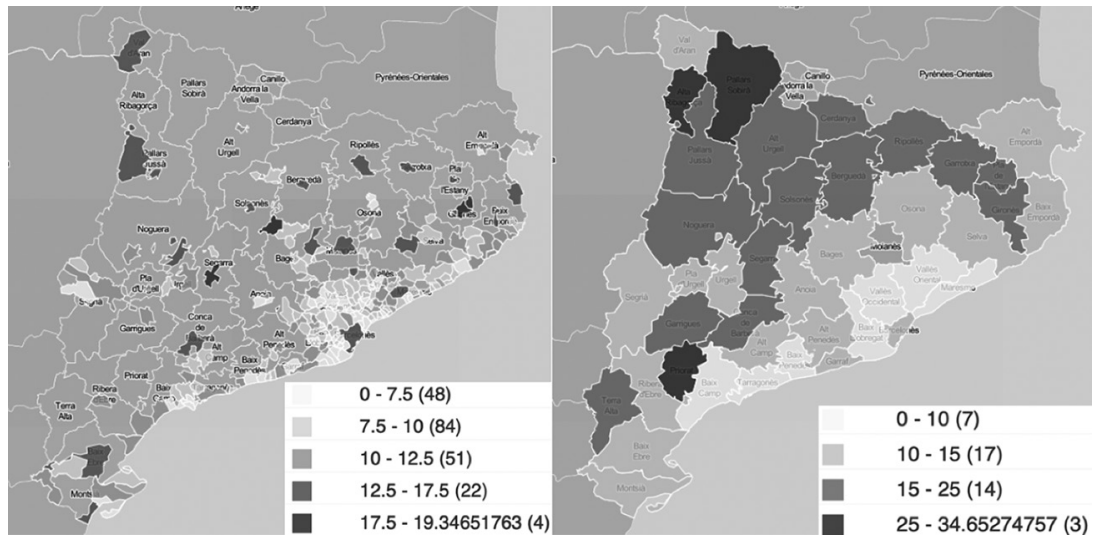


Según muestra la Figura 3, el Área Metropolitana de Barcelona es donde la densidad asociativa es menor, probablemente en parte por el hecho de ser ciudades dormitorio y por el efecto de la capitalidad de Barcelona, que puede favorecer que ciertas asociaciones y federaciones se establezcan allí. La falta de municipios mayores de 5000 habitantes en las comarcas interiores no permite visualizar gráficamente las diferencias, si bien se intuye una densidad más elevada en los municipios del centro y el norte. A nivel comarcal la densidad asociativa es claramente más elevada en las comarcas de los Pirineos y el Prepirineo, así como en el Priorat.

Las donaciones de sangre, como muestra la Figura 4, tienen una volatilidad elevada, que va de las 6,48 donaciones por 1000 habitantes de Cubelles a las 89,85 de Centelles. Destacan los municipios de las provincias de Girona, Lleida y Tarragona, estando los valores más grandes y pequeños repartidos por toda la geografía, de modo que no se pueden extraer conclusiones claras. En el mapa de la derecha se pueden apreciar unos mayores niveles de donaciones en las comarcas del tercio oeste y del noreste del territorio.

\section{Figura 4. Índice medio de donaciones de sangre municipa- les y comarcales, 2012-2018 (en donaciones por 1.000 hab.)}

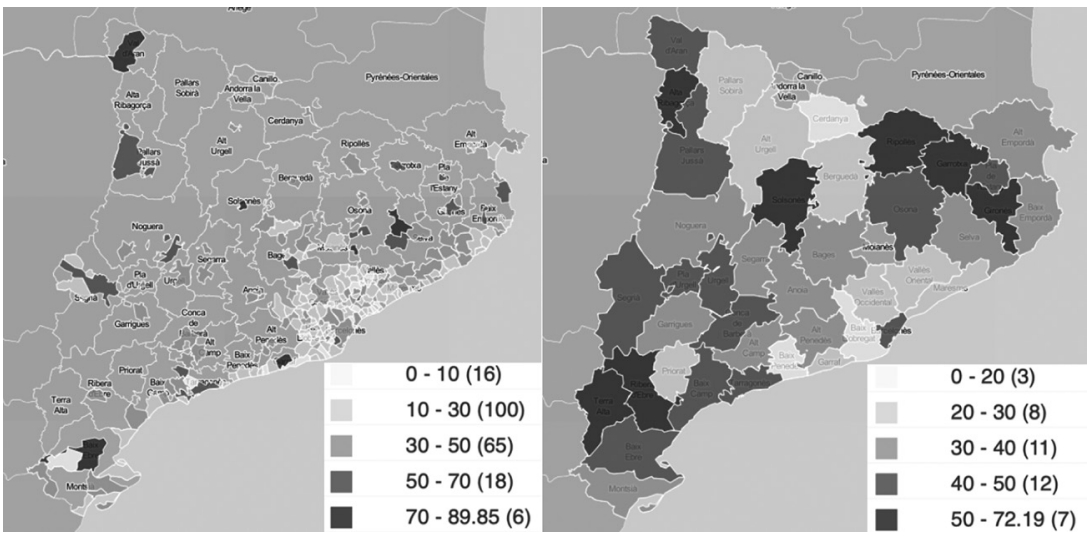

Los fundamentos teóricos del modelo están basados en Rupasingha et al. y Becker, explicados más extensamente en su artículo sobre la producción de capital social en los condados de los Estados Unidos, y se parte de un modelo de toma de decisiones en el cual el individuo elige cuánto capital social producir, y esto depende del coste de oportunidad de asignar tiempo y recursos y de los beneficios marginales de un mayor capital social (Becker, 1965; Rupasingha et al., 2006). Se parte de un hogar representativo de un municipio o comarca $i$, con la función de utilidad cuasicóncava siguiente (1), que depende del consumo $\left(C_{j}\right)$ y del capital social $\left(S K_{j}\right)$ :

1.- Ver el enlace a todos los mapas interactivos en línea en el anexo. 


$$
U_{i}=U\left(C_{i}, S K_{i}\right) \text { donde } i=1, \ldots, n
$$

La ecuación (2) presenta la lagrangiana para poder resolver el problema de maximización de la utilidad del hogar:

$$
L=U\left(C\left(x_{c} t_{c}\right), S K\left(x_{s}, t_{s}, E_{i}, R_{j}\right)\right)+\lambda\left[w_{i}\left(T-t_{c}-t_{s}\right)-\left(p_{x} x_{c}+p_{s} x_{s}\right)\right]
$$

donde el consumo y el capital social dependen de los bienes de mercado $\left(x_{c}\right)$ y la asignación de tiempo para su producción $\left(t_{c}\right)$, y de los bienes de capital social $\left(x_{s}\right)$, la asignación de tempo para su producción $\left(t_{s}\right)$, las características propias del hogar $\left(E_{j}\right)$ y las características de los otros hogares de la comunidad $\left(R_{j}\right)$ respectivamente. Las restricciones son temporales $((T)$ es el tiempo disponible por periodo y $\left(t_{1}\right)$ es el dedicado a trabajar) y de presupuesto, con $\left(p_{x}\right),\left(p_{s}\right)$ y $\left(w_{j}\right)$ describiendo el precio del bien de mercado, el precio de los bienes de capital social y el salario del hogar, respectivamente. Aplicando las condiciones de primer orden se encuentra el punto donde el hogar elige un nivel óptimo de capital social $\left(x_{s}\right)$ estrictamente positivo, cuando la utilidad marginal del bien de capital social es superior al coste de oportunidad marginal, como refleja la ecuación (3).

$$
Y^{*}=\frac{\left(U^{\prime} S K^{\prime}(\cdot)\right)}{\lambda}-p_{S}>0
$$

Los términos observados y los controles se incorporan al modelo de capital social a los municipios $y$ comarcas de Cataluña (vector $x$ de la ecuación (4)) para cada una de las variables estudiadas:

$$
Y_{i}^{*}=\beta x_{i}+u_{i}
$$

donde $u_{j}$ es el término de error. Este modelo se ha estimado mediante Stata 12 por mínimos cuadrados ordinarios (MCO) en el caso de datos de corte transversal. En el caso de las donaciones de sangre, la posibilidad de estimar modelos con datos de panel lleva a una disyuntiva entre usar una estimación de efectos fijos o una de efectos aleatorios, con sus ventajas e inconvenientes. Por un lado, una regresión con efectos fijos produce una estimación consistente de los efectos de las variables que varían en el tiempo, pero no de las invariables, pues son omitidas. En cambio, una regresión con efectos aleatorios las tiene en cuenta, pero su estimación puede no ser consistente. La decisión sobre qué modelo usar depende de la relevancia de las variables invariables en el tiempo, y en el presente caso es importante conocer el efecto de factores como la educación, la densidad asociativa y el porcentaje de viviendas en propiedad en los niveles de donación de sangre, por lo que se ha preferido asumir el riesgo con una estimación con efectos aleatorios usando las donaciones de sangre de los siete años disponibles como variable dependiente, así como la renta, población, densidad de población, inmigración, movilidad geográfica y porcentaje de mayores de 65 años como las otras variables con siete periodos disponibles. En el estudio de Rupasingha et al. en el cual se basa este trabajo, los autores justifican su elección de un modelo de efectos aleatorios con un argumento similar (Rupasingha et al., 2006), de modo que se ha preferido poder incluir todas las estimaciones. 


\section{Resultados}

Las tablas 3 a 5 muestran los resultados de las regresiones realizadas con las tres variables consideradas como parte del capital social. Concretamente, la Tabla 3 contiene las correspondientes a la participación electoral en los municipios y las comarcas. A pesar de no controlar por la oferta política se puede apreciar una $R^{2}$ moderadamente elevada a nivel municipal, y ligeramente inferior a nivel comarcal, en las regresiones (1) y (2) respectivamente. En los municipios destaca la relación positiva de la renta y la densidad asociativa con la participación en las urnas, así como el impacto negativo de la movilidad geográfica. En menor medida, el porcentaje de mayores de 65 años afecta negativamente a la participación, así como el porcentaje de inmigración y el tamaño de la población. En las comarcas, únicamente la densidad asociativa tiene una significación superior al $90 \%$.

La Tabla 4 muestra las variables relacionadas con la densidad asociativa. En la regresión (3) son relevantes los coeficientes positivos del porcentaje de habitantes municipales con educación terciaria, y de la altitud. En cambio, la renta, la densidad de población y el porcentaje de viviendas en propiedad parecen perjudicar el establecimiento de asociaciones y federaciones. Asimismo, se ha encontrado una relación no lineal entre el porcentaje de mayores de 65 años y la variable dependiente, siendo cuadrática con un mínimo en alrededor del 13,3\% de población anciana. La mediana de esta variable está situada en $16,49 \%$, de modo que el efecto es mayoritariamente positivo, en la parte ascendente de la parábola. En las comarcas, la regresión (4) muestra que el efecto de tener una población más envejecida es claramente positivo, y solo resulta levemente significativo el coeficiente negativo de la densidad de población. 


\section{Tabla 3. Determinantes de la participación electoral}

\begin{tabular}{|c|c|c|}
\hline & (1) & $(2)$ \\
\hline Participación electoral & Municipios & Comarcas \\
\hline \multirow[t]{2}{*}{ eduter } & 0.0425 & -0.122 \\
\hline & $(0.0375)$ & $(0.127)$ \\
\hline \multirow[t]{2}{*}{ rentam } & $0.723^{\star \star \star}$ & -0.178 \\
\hline & $(0.114)$ & $(0.283)$ \\
\hline \multirow[t]{2}{*}{ poblacionm } & $-3.05 e-05^{\star * *}$ & $2.80 \mathrm{e}-07$ \\
\hline & $(7.51 \mathrm{e}-06)$ & $(1.26 \mathrm{e}-06)$ \\
\hline \multirow[t]{2}{*}{ logdpm } & 0.106 & -0.00736 \\
\hline & $(0.150)$ & $(0.271)$ \\
\hline \multirow[t]{2}{*}{ asoc } & $0.162^{\star *}$ & $0.278^{\star \star *}$ \\
\hline & $(0.0628)$ & $(0.0784)$ \\
\hline \multirow[t]{2}{*}{ inm } & $-0.0612^{* *}$ & $-0.164^{*}$ \\
\hline & $(0.0277)$ & $(0.0858)$ \\
\hline \multirow[t]{2}{*}{ movilidadm } & $-0.511^{\star * \star}$ & $-0.379^{*}$ \\
\hline & $(0.0845)$ & $(0.214)$ \\
\hline \multirow[t]{2}{*}{ maym } & $-0.115^{\star \star}$ & 0.0295 \\
\hline & $(0.0573)$ & $(0.144)$ \\
\hline \multirow[t]{2}{*}{ propiedad } & -0.0257 & 0.0479 \\
\hline & $(0.0245)$ & $(0.0590)$ \\
\hline \multirow[t]{2}{*}{ Constant } & $62.18^{\star \star \star}$ & $71.42^{\star \star \star}$ \\
\hline & (3.0568) & $(8.492)$ \\
\hline Observaciones & 199 & 41 \\
\hline R-cuadrado & 0.641 & 0.593 \\
\hline
\end{tabular}

Errores estándar entre paréntesis

${ }^{* * *} p<0.01,{ }^{* *} p<0.05,{ }^{*} p<0.1$ 


\section{Tabla 4. Determinantes de la densidad asociativa}

\begin{tabular}{|c|c|c|}
\hline & (3) & (4) \\
\hline Densidad asociativa & Municipios & Comarcas \\
\hline \multirow[t]{2}{*}{ eduter } & $0.129^{\star \star *}$ & 0.301 \\
\hline & $(0.0420)$ & $(0.281)$ \\
\hline \multirow[t]{2}{*}{ rentam } & $-0.293^{* *}$ & 0.788 \\
\hline & $(0.131)$ & $(0.624)$ \\
\hline \multirow[t]{2}{*}{ poblacionm } & $2.36 \mathrm{e}-06$ & $-3.42 \mathrm{e}-06$ \\
\hline & $(8.36 e-06)$ & $(2.78 \mathrm{e}-06)$ \\
\hline \multirow[t]{2}{*}{ logdpm } & $-0.444^{\star \star \star}$ & $-1.0446^{*}$ \\
\hline & $(0.169)$ & $(0.583)$ \\
\hline \multirow[t]{2}{*}{ inm } & $-10.87 e-05$ & -0.00501 \\
\hline & $(0.0314)$ & $(0.193)$ \\
\hline \multirow[t]{2}{*}{ movilidadm } & 0.0430 & 0.255 \\
\hline & $(0.0950)$ & $(0.481)$ \\
\hline \multirow[t]{2}{*}{ maym } & $-0.908^{\star *}$ & $0.993^{* \star *}$ \\
\hline & $(0.435)$ & $(0.273)$ \\
\hline \multirow[t]{2}{*}{ maym² $^{2}$} & $0.0340^{* *}$ & \\
\hline & $(0.0131)$ & \\
\hline \multirow[t]{2}{*}{ propiedad } & $-0.0918^{\star * *}$ & -0.193 \\
\hline & $(0.0265)$ & $(0.129)$ \\
\hline \multirow[t]{2}{*}{ logaltitud } & $0.458^{* \star *}$ & \\
\hline & $(0.136)$ & \\
\hline \multirow[t]{2}{*}{ Constant } & $23.76^{* \star *}$ & -6.11 \\
\hline & $(4.776)$ & (19.121) \\
\hline Observaciones & 199 & 41 \\
\hline R-cuadrado & 0.436 & 0.485 \\
\hline
\end{tabular}

Errores estándar entre paréntesis

${ }^{* \star *} p<0.01,{ }^{* *} p<0.05,{ }^{*} p<0.1$ 
A continuación, la Tabla 5 muestra los determinantes de las donaciones de sangre. Según la regresión (5), el tamaño de los municipios y la densidad asociativa tienen un efecto positivo en las donaciones de sangre, mientras que una mayor densidad de población, movilidad residencial o porcentaje de viviendas en propiedad afectan negativamente al número de donaciones realizadas. La renta familiar disponible media parece tener un efecto máximo en los $15.390 €$, un valor cercano a la mediana, de modo que el impacto puede ir en ambas direcciones. La regresión (6) muestra los resultados de la regresión del panel con datos de los siete periodos que van de 2012 a 2018 para la renta familiar disponible media, población, densidad de población, porcentaje de inmigración, movilidad residencial y porcentaje de mayores de 65 años. Se mantienen los signos de los coeficientes del tamaño de la población, densidad asociativa y régimen de propiedad, mientras que el efecto de la educación terciaria y la población anciana se convierte en significativo. Por el contrario, la renta, la densidad de población y la movilidad geográfica pierden su significación estadística.

En las comarcas, las regresiones (7) y (8) siguen la misma idea. En la regresión (7), con datos de corte transversal, el impacto de la educación terciaria es positivo, pero solamente con un valor $p$ inferior a 0.1 , mientras que la densidad asociativa y el coeficiente de movilidad siguen formas cuadráticas, con máximo en $40 \%$ (que al ser superior a todos los valores de la base de datos implica un efecto positivo) y un mínimo en 10,87 asociaciones por 1000 habitantes respectivamente. Usando los datos de panel, la regresión (8) muestra una mayor significación estadística que en la anterior regresión, y un efecto mínimo cuando la población con estudios superiores es del 24,28\%. La densidad asociativa también aumenta su significación, y su efecto máximo está situado en 20,46 asociaciones por 1000 habitantes. Por su parte, la movilidad geográfica mantiene sus signos, pero su valor $p$ pasa a ser superior a 0.05 . El efecto de la población anciana se mantiene negativo y se convierte en significativo, aunque solamente al $90 \%$. 


\section{Tabla 5. Determinantes de las donaciones de sangre}

\begin{tabular}{|c|c|c|c|c|}
\hline \multirow[t]{2}{*}{ Donaciones } & (5) & (6) & (7) & (8) \\
\hline & Municipios & Panel municipios & Comarcas & Panel comarcas \\
\hline \multirow[t]{2}{*}{ eduter } & -0.104 & $-0.386^{* *}$ & $1.194^{\star}$ & $-9.129^{\star *}$ \\
\hline & $(0.256)$ & $(0.173)$ & $(0.641)$ & (3.974) \\
\hline \multirow[t]{2}{*}{ eduter $^{2}$} & & & & $0.188^{* *}$ \\
\hline & & & & $(0.0815)$ \\
\hline \multirow[t]{2}{*}{ rentam } & $7.143^{*}$ & -0.203 & -2.244 & -0.416 \\
\hline & (3.855) & $(0.306)$ & $(1.406)$ & $(0.714)$ \\
\hline \multirow[t]{2}{*}{ rentam ${ }^{2}$} & $-0.232^{* *}$ & & & \\
\hline & $(0.112)$ & & & \\
\hline \multirow[t]{2}{*}{ poblacionm } & $9.38 \mathrm{e}-05^{*}$ & $1.56 \mathrm{e}-04^{\star * *}$ & $-9.24 e-06$ & $-1.23 e-07$ \\
\hline & $(5.19 e-05)$ & $(4.81 e-05)$ & $(6.83 e-06)$ & $(6.57 e-06)$ \\
\hline \multirow[t]{2}{*}{ logdpm } & $-2.376^{\star *}$ & $-2.725^{\star \star \star}$ & -0.237 & -1.880 \\
\hline & $(1.0176)$ & $(0.998)$ & $(1.356)$ & $(1.192)$ \\
\hline \multirow[t]{2}{*}{ asoc } & $1.253^{\star \star \star}$ & $1.489^{\star \star \star}$ & $4.565^{\star \star}$ & $6.835^{\star \star \star}$ \\
\hline & $(0.434)$ & $(0.427)$ & $(1.807)$ & $(1.716)$ \\
\hline \multirow[t]{2}{*}{$\operatorname{asoc}^{2}$} & & & $-0.114^{* *}$ & $-0.167^{\star \star \star}$ \\
\hline & & & $(0.0420)$ & $(0.0414)$ \\
\hline \multirow[t]{2}{*}{$\mathrm{inm}$} & 0.00438 & -0.0898 & 0.347 & -0.305 \\
\hline & $(0.209)$ & $(0.119)$ & $(0.446)$ & $(0.309)$ \\
\hline \multirow[t]{2}{*}{ movilidadm } & $-1.679^{\star \star \star}$ & 0.0517 & $-23.495^{\star \star}$ & $-1.131^{*}$ \\
\hline & $(0.575)$ & $(0.184)$ & (8.881) & $(0.579)$ \\
\hline \multirow[t]{2}{*}{ movilidadm² } & & & $1.0809^{* *}$ & $0.0240^{*}$ \\
\hline & & & $(0.467)$ & $(0.0132)$ \\
\hline \multirow[t]{2}{*}{ maym } & -0.261 & $-0.570^{* * *}$ & -0.108 & $-1.0254^{\star}$ \\
\hline & $(0.394)$ & $(0.172)$ & $(0.924)$ & $(0.564)$ \\
\hline \multirow[t]{2}{*}{ propiedad } & $-0.467^{\star \star \star}$ & $-0.542^{\star \star \star}$ & 0.281 & 0.516 \\
\hline & $(0.167)$ & $(0.146)$ & $(0.323)$ & $(0.322)$ \\
\hline \multirow[t]{2}{*}{ Constant } & 32.11 & $90.35^{\star \star \star}$ & 102.70 & 95.71 \\
\hline & $(39.400)$ & (15.156) & $(69.678)$ & $(67.207)$ \\
\hline Observaciones & 199 & 1,393 & 41 & 287 \\
\hline R-cuadrado & 0.381 & & 0.618 & \\
\hline Número de id & & 199 & & 41 \\
\hline
\end{tabular}

Errores estándar entre paréntesis

${ }^{* * *} p<0.01,{ }^{* *} p<0.05,{ }^{*} p<0.1$ 
Para sintetizar los resultados y siguiendo a Rupasingha et al. (2006), hemos creído oportuno elaborar un índice de capital social a partir de las variables estudiadas. Otros autores lo han hecho a nivel de país, como bien recogen Bellostas Pérez-Grueso et al. en su estudio sobre el capital social y las organizaciones no lucrativas (Bellostas Pérez-Grueso et al., 2007). En nuestro caso esto se ha conseguido realizando un análisis de los componentes principales de la participación electoral media, la densidad asociativa, y las donaciones de sangre medias. La medida de adecuación muestral de Kaiser-Meyer-Olkin compara la magnitud de los coeficientes de correlación observados con la de los coeficientes de correlación parciales, y un valor de 0,52 indica que es mínimamente adecuado realizar este análisis a nivel comarcal. A nivel municipal el valor es de 0,495, cercano al 0,5 mínimo aconsejable, de modo que se ha procedido igualmente.

Se ha considerado como índice de capital social al primer componente principal que se obtiene de las combinaciones lineales entre las tres variables, pues es el único con el valor propio superior a 1, y explica un $48 \%$ de la variación total en el caso de los municipios. El peso de cada variable en el índice no es necesariamente el mismo, sino que se le asigna una proporción distinta en la combinación lineal según el componente principal y la distribución de las variables. En el caso del primer componente principal del índice municipal, la densidad asociativa $(0,71)$ y las donaciones de sangre $(0,69)$ tienen un peso claramente mayor que la participación electoral $(0,18)$. En las comarcas, el primer componente principal explica el $57 \%$ de la variación en los datos, con un peso importante de la participación electoral $(0,67)$ y la densidad asociativa $(0,63)$ en los valores de la combinación lineal, y menor el de las donaciones de sangre $(0,39)$.

La Figura 5 muestra la distribución espacial del índice para los dos niveles estudiados, logrando los niveles más elevados en municipios menores de 30.000 habitantes del interior, con las excepciones de Girona y Tortosa (mayor población), y Torroella de Montgrí (situado en la costa). El resto de municipios con mayor capital social son: Cardona y Centelles en la provincia de Barcelona; Arbúcies, Banyoles, Celrà, Ripoll y Sant Hilari Sacalm en la provincia de Girona; Cervera, Solsona, Tremp y Vielha en la provincia de Lleida y Montblanc en la de Tarragona. Los niveles más bajos, en cambio, corresponden a Calafell, Canovelles, Cornellà de Llobregat, Rubí, Sant Adrià de Besòs, Sant Boi de Llobregat, Santa Coloma de Gramenet, Vallirana y Viladecans. Ocho de los nueve municipios pertenecen a la provincia de Barcelona, cinco a su área metropolitana y otros cinco tienen más de 60.000 habitantes, validando la idea de las ciudades dormitorio como pobres en capital social. En las comarcas, el patrón es menos claro, pero sí que resultan evidentes los menores niveles en la costa y el área metropolitana de Barcelona. 


\section{Figura 5. Índice de capital social municipal y comarcal (a mayor valor, mayor capital social)}
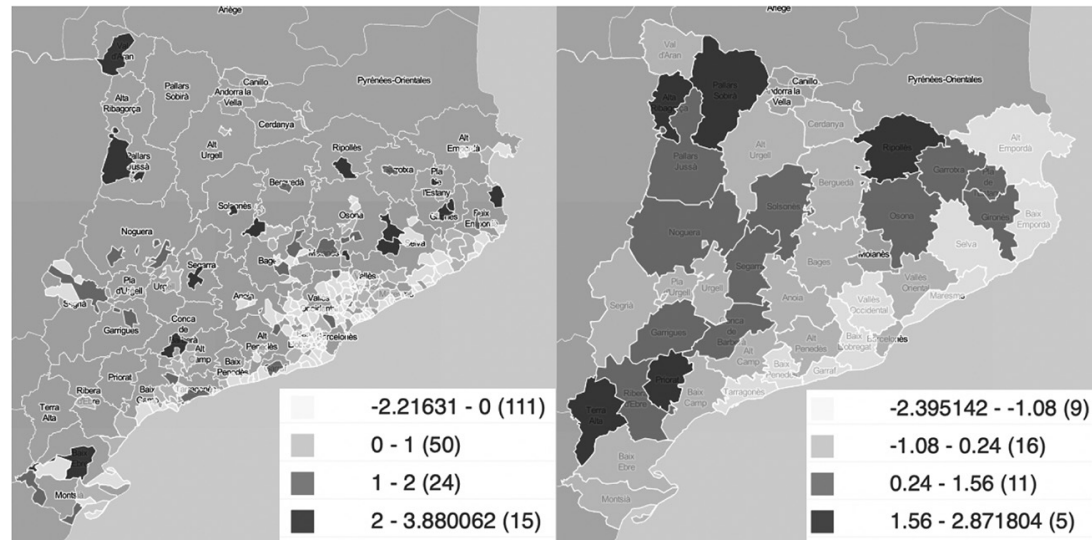

\section{Discusión}

Los resultados muestran una cantidad relativamente elevada de variables significativas que siguen los patrones esperados, de modo que los resultados serían válidos internamente, sobretodo a nivel municipal, aunque haría falta acotar la definición de capital social para dotarla de una mayor validez y comparabilidad externa.

La evidencia sobre la relación entre vivienda en propiedad y participación política y comunitaria muestra que esta es generalmente positiva (Dipasquale \& Glaeser, 1999; Glaeser et al., 2002; McCabe, 2013). Los resultados presentados en las tablas anteriores, en cambio, muestran coeficientes negativos y significativos excepto en la participación electoral. Puede ser que el efecto varíe según la renta y según el tipo de estrategia de identificación seguida, pues Engelhardt et al. encuentran efectos nulos o negativos al estudiar el efecto de la propiedad de la vivienda en la participación del segmento de población con bajos ingresos y mediante el uso de variables instrumentales (Engelhardt, Eriksen, Gale, \& Mills, 2010). Rupasingha et al. encontraron un efecto negativo y persistente de la propiedad de la vivienda (Rupasingha et al., 2006). En el presente trabajo, con la limitación que supone obtener 
datos a nivel municipal y comarcal, y aún controlando por la movilidad geográfica, pues es relevante tenerla en cuenta al estar asociada al tipo de régimen de tenencia (Dipasquale \& Glaeser, 1999), no ha desaparecido el efecto negativo, tampoco usando solamente la participación en elecciones locales como variable dependiente, al tener posiblemente un impacto más elevado de la vivienda en propiedad que el resto de comicios (McCabe, 2013).

El coeficiente del porcentaje de población extranjera es negativo en el caso de la participación electoral media (Tabla 3), coherente si se tienen en cuenta las limitaciones en el derecho a voto que afectan a parte de este colectivo. Para las otras variables la inmigración no presenta ninguna tendencia clara. Es posible que para poder apreciar un mayor efecto se tenga que poner el foco en el estudio de los barrios y en la distribución de la población extranjera en los mismos. En Estados Unidos el impacto es relevante y negativo (Alesina \& La Ferrara, 2000), pues crea aislamiento social (Putnam, 2007). Recientemente también se han descrito posibles interacciones complejas entre la diversidad racial y el capital social y la desigualdad de renta en los condados de los Estados Unidos (Paarlberg, Hoyman, \& McCall, 2018). En Europa, Tolsma et al. no han encontrado ningún impacto claro de la inmigración en la cohesión social de los municipios y barrios holandeses (Tolsma, Meer, \& Gesthuizen, 2009).

La educación tiene un efecto muy limitado en los resultados del trabajo, con coeficientes mayoritariamente positivos pero pequeños y sin ser significativos en muchos casos. La imposibilidad de conseguir un instrumento fiable para medir la educación provoca que su estimación tampoco lo sea. La literatura es muy clara al respecto: la educación es clave para aumentar los niveles de capital social y el impacto de la misma es positivo (Jian, Maasen van den Brink, \& Groot, 2009).

La medida de movilidad geográfica basada en las migraciones internas afecta negativamente y de manera significativa al capital social, especialmente en los municipios y exceptuando los efectos en la densidad asociativa. La relación negativa sigue la teoría según la cual una mayor movilidad residencial impide crear vínculos profundos, y va en la línea de la evidencia presentada por Rupasingha et al., si bien está medida de diferente forma (Rupasingha et al., 2006).

La renta familiar disponible bruta no muestra un efecto claro aunque se prevé una relación positiva, pues el hecho de tener bajos ingresos puede implicar más horas de trabajo para asegurar más ingresos, dejando menos tiempo para la participación cívica (Rupasingha et al., 2006). Solo tiene un claro impacto positivo en la participación electoral municipal, y en la parte ascendente del efecto cuadrático de las donaciones de sangre. En los municipios más pobres el efecto de la renta es mayor.

Los efectos de la proporción de mayores de 65 años en un municipio son generalmente negativos, y positivos y significativos en el caso de la densidad asociativa. Los signos negativos irían en la línea descrita por la evidencia que encuentra una relación de $U$ invertida entre la edad y el capital social (Glaeser et al., 2002), de modo que un mayor número de personas mayores en la ciudad podría hacer 
decrecer sus niveles. La jubilación también puede afectar negativamente a la acumulación de capital social conseguida durante el tiempo de actividad laboral, con relaciones complejas entre entorno laboral, no laboral y género (McDonald \& Mair, 2010). Finalmente, la densidad asociativa presenta coeficientes positivos 0 cuadráticos tanto en la participación electoral como en las donaciones de sangre, y generalmente significativos, lo que muestra una relación clara con otros atributos del capital social, permitiendo usar ambos conceptos indistintamente.

Es preciso tener en cuenta que la medida de movilidad presenta una posible desventaja, pues se puede dar el caso de municipios que estén perdiendo población porque sus habitantes emigran por falta de oportunidades, y esta decadencia se interpreta de la misma manera que una ciudad dinámica donde hay una gran rotación de personas debido a migraciones internas, de modo que se pierde cierta fiabilidad.

Puede haber un efecto capitalidad en el caso de la densidad asociativa y las donaciones de sangre, pues en el primer caso ciertas asociaciones pueden elegir establecerse en la capital de comarca, aunque operen en diferentes municipios, por una cuestión lógica de escala. En el caso de las donaciones también es lógico que el banco de sangre concentre sus campañas de donación en municipios de más de 5.000 habitantes y capitales de comarca. En relación con esto, sería importante evaluar los determinantes del rendimiento en las donaciones de sangre, pues a parte del número de donaciones al año también es relevante tener en cuenta el número de campañas realizadas en cada municipio para poder dar más validez a los resultados.

No ha sido posible elaborar instrumentos viables para la educación ni la concentración de renta, siguiendo a Rupasingha et al., pero tampoco para otras variables como el régimen de propiedad, debido a la falta de datos disponibles tanto municipales como comarcales sobre gasto en salud y educación, años de escolarización, grupos de edad, tamaño familiar o tipo de distribución urbana. Es importante disponer de este 0 algún otro método para identificar correctamente el impacto de al menos estas variables.

Se disponía de datos de desigualdad y concentración municipal de la renta para el año 2007 provenientes de la Fundación de Estudios de Economía Aplicada (Fedea), pero desafortunadamente se ha descartado su uso debido a su antigüedad, a pesar de ser una variable muy relevante tanto para conocer el impacto de la desigualdad de renta en el capital social (Alesina \& La Ferrara, 2000; Gould \& Hijzen, 2016) como en sus consecuencias, por ejemplo, en la salud (Aida et al., 2011; Gold, Kennedy, Connell, \& Kawachi, 2002; Kawachi, Kennedy, Lochner, \& Prothrow-Stith, 1996; Morgan \& Haglund, 2009).

De cara a futuras investigaciones, resultaría interesante intentar aproximar la causalidad y el procedimiento de producción del capital social, por ejemplo, cuánto tarda un cierto cambio en las variables explicativas a afectar a los niveles de capital social, o bien si se produce algún ciclo vital 
(Glaeser et al., 2002). Resultaría útil poder determinar si en Cataluña también se produce el fenómeno de descapitalización detectado en Bowling Alone (Putnam, 2000). En la serie temporal del conjunto de Cataluña las donaciones de sangre medias son ligeramente menores en esta década que en la anterior, aunque se tendría que profundizar en los otros determinantes y en el abanico de causas. El hecho de diferenciar entre asociacionismo tipo Olson y tipo Putnam también podría ayudar a descontaminar esta variable y por lo tanto a mejorar la validez de los resultados (Knack \& Keefer, 1997; Rupasingha et al., 2006). Por otro lado, los estudios de caso en los municipios con mayores niveles de capital social podrían dar pistas sobre las características compartidas que permiten dichos niveles, así como los análisis a nivel de barrio para ciudades grandes o con grandes diferencias educativas o de renta.

\section{Conclusiones}

El presente artículo ofrece tres contribuciones principales. En primer lugar, mediante el uso de las tres variables asociadas al capital social, se han podido identificar algunos de sus principales determinantes tanto en los municipios como en las comarcas de Cataluña. También se ha observado un comportamiento a veces diferenciado a nivel comarcal. En segundo lugar, se ha podido comprobar como un índice de capital social a partir de las tres variables estudiadas resulta útil para fines comparativos entre municipios o comarcas, pero el problema de la medición del concepto está lejos de resolverse debido a la dificultad de su cuantificación. En tercer lugar, la representación geográfica de los datos ha permitido observar unos leves patrones geográficos en la distribución del capital social y las tres variables asociadas estudiadas, que podrían ayudar a explicar las diferencias en los niveles observados.

A pesar de esto, el presente estudio deja más preguntas que respuestas, pues la cuestión del método universal de medición indirecta del capital social está lejos de resolverse, y se abren muchos campos para futuras investigaciones tanto a nivel local como en el caso particular de Cataluña. Algunos de los resultados no coinciden con los predominantes en la evidencia existente, pero es probable que pudiendo instrumentar ciertas variables y disponiendo de datos menos agrupados y más frecuentes los resultados estén más en sintonía con la evidencia, de modo que el caso de Cataluña en principio no representaría una excepción. Asimismo, se deben afinar las técnicas para capturar los efectos para los cuales no se dispone de mediciones a escala municipal, así como para tener en cuenta las características geográficas. Este ha sido un intento de explicar aquellas diferencias intangibles entre municipios, y quizás entre comarcas, que no tienen un origen claro pero que son las que, con el tiempo, dan cohesión social a los territorios. 


\section{Agradecimientos}

Es preciso agradecer las valiosas aportaciones de Claudia Rei (University of Warwick) durante la fase inicial del estudio, así como al Banco de Sangre y Tejidos de Cataluña por la amable provisión de datos históricos de donaciones de sangre, al Instituto de Estudios Regionales y Metropolitanos de Barcelona (Universitat Autònoma de Barcelona) por la generosa provisión de datos de sus encuestas sobre relaciones vecinales y cohesión urbana a pesar de no haber sido usadas finalmente en el estudio, a Pol Guillaumes y Adrià Mateu por sus comentarios y a los evaluadores anónimos de la Revista CIRIEC-España por sus acertadas propuestas de mejora. Finalmente, a Khalid Khayati (Linköpings Universitet) hay que agradecerle sus influencias interdisciplinarias de la Sociología y la Ciencia Política que de alguna manera han acabado inspirando este trabajo, y a Vicente Ortún (Universitat Pompeu Fabra) hay que agradecerle muy especialmente su confianza, apoyo, aportaciones y experiencia que han hecho posible y enriquecido significativamente esta obra.

\section{Referencias}

ADLER, P.S. \& KWON, S. (2002): "Social capital: prospects for a new concept", The Academy of Management Review, 27(1), 17-40. DOI: 10.5465/AMR.2002.5922314.

AIDA, J., KONDO, K., KONDO, N., WATT, R.G., SHEIHAM, A. \& TSAKOS, G. (2011): "Income inequality, social capital and self-rated health and dental status in older Japanese", Social Science and Medicine, 73(10), 1561-1568. DOI: 10.1016/j.socscimed.2011.09.005.

ALESINA, A. \& LA FERRARA, E. (2000): "Participation in heterogeneous communities", Quarterly Journal of Economics, 115(3), 847-904. DOI: 10.1162/003355300554935.

ARROW, K.J. (2000): "Observations on social capital". In P. Dasgupta \& I. Serageldin (Eds.), Social capital: A multifaceted perspective, Washington DC: World Bank, 3-5. DOI: 10.1596/978-0-82135004-1.

BECKER, G. (1965): "A theory of the allocation of time", The Economic Journal, 75(299), DOI: $10.2307 / 2228949$.

BEKKERS, R. \& VELDHUIZEN, I. (2008): "Geographical differences in blood donation and philanthropy in the Netherlands - What role for social capital?", Tijdschrift Voor Economische En Sociale Geografie, 99(4), 483-496. DOI:10.1111/j.1467-9663.2008.00483.x. 
BELLOSTAS PÉREZ-GRUESO, A., MARCUELLO SERVÓS, C. \& MONEVA ABADÍA, J.M. (2007): Capital Social y Organizaciones No Lucrativas en España: El Caso de las ONGD (C. Marcuello Servós, Ed.), Bilbao: Fundación BBVA.

BOURDIEU, P. (1986): "The forms of capital". In M. Granovetter \& R. Swedberg (Eds.), The Sociology of Economic Life, New York: Routledge. DOI: 10.4324/9780429494338.

BUONANNO, P., MONTOLIO, D. \& VANIN, P. (2009): "Does social capital reduce crime?", Journal of Law and Economics, 52(1), 145-170. DOI: 10.1086/595698.

COLEMAN, J.S. (1988): "Social capital in the creation of human capital", The American Journal of Sociology, 94(Supplement: Organizations and Institutions: Sociological and Economic Approaches to the Analysis of Social Structure), S95-S120. DOI: 10.1086/228943.

DE NIEVES NIETO, C. \& BRIONES PEÑALBER, A.J. (2009): "Las empresas de economía social y su relación con las instituciones: colaboración con la universidad en asuntos medioambientales", CIRIEC-España, Revista de Economía Pública, Social y Cooperativa, 65, 85-111.

DIPASQUALE, D. \& GLAESER, E.L. (1999): "Incentives and social capital: are homeowners better citizens?", Journal of Urban Economics, 45(2), 354-384. DOI: 10.1006/juec.1998.2098.

ENGBERS, T.A., THOMPSON, M.F., \& SLAPER, T. (2017): "Theory and measurement in social capital research", Social Indicators Research, 132(2), 537-558. DOI: 10.1007/s11205-016-1299-0.

ENGELHARDT, G.V., ERIKSEN, M.D., GALE, W.G., \& MILLS, G.B. (2010): "What are the social benefits of homeownership? Experimental evidence for low-income households" Journal of Urban Economics, 67(3), 249-258. DOI: 10.1016/j.jue.2009.09.010.

FISCHER, C.S. (2001): "Bowling alone: what's the score?", Social Networks, 27(2), 155-167, DOI: 10.1016/j.socnet.2005.01.009.

GLAESER, E.L., LAIBSON, D. \& SACERDOTE, B. (2002): "An economic approach to social capital", The Economic Journal, 112(483), F437-F458. DOI: 10.1111/1468-0297.00078.

GLAESER, E.L., SCHEINKMAN, J.A. \& SOUTTER, C. L. (1999): "What is social capital? The determinants of trust and trustworthiness", NBER Working Paper Series, 7216. DOI: 10.3386/w7216.

GOLD, R., KENNEDY, B., CONNELL, F. \& KAWACHI, I. (2002): "Teen births, income inequality, and social capital: Developing an understanding of the causal pathway", Health and Place, 8(2), 77-83. DOI: 10.1016/S1353-8292(01)00027-2.

GOULD, E.D. \& HIJZEN, A. (2016): "Growing apart, losing trust? The impact of inequality on social capital", IMF Working Papers, 16(176). DOI: 10.5089/9781475529487.001.

GUISO, L., SAPIENZA, P. \& ZINGALES, L. (2011): "Civic capital as the missing link". In J. Benhabib, A. Bisin, \& M. Jackson (Eds.), Handbook of Social Economics, Amsterdam: North-Holland, 417-480. DOI: 10.1016/B978-0-444-53187-2.00010-3. 
HANIFAN, L.J. (1916): "The rural school community center", The Annals of the American Academy of Political and Social Science, 67(1), 130-138. DOI: 10.1177/000271621606700118.

JIAN, H., MAASEN VAN DEN BRINK, H. \& GROOT, W. (2009): "A meta-analysis of the effect of education on social capital", Economics of Education Review, 28(4), 454-464. DOI: 10.1016/j. econedurev.2008.03.004.

KAWACHI, I., KENNEDY, B.P., LOCHNER, K. \& PROTHROW-STITH, D. (1996): "Social capital, income inequality, and mortality", American Journal of Public Health, 87(9), 1491-1498. DOI: 10.2105/ajph.87.9.1491.

KENWORTHY, L. (1997): "Civic engagement, social capital, and economic cooperation", American Behavioral Scientist, 40(5), 645-656. DOI: 10.1177/0002764297040005010.

KNACK, S. \& KEEFER, P. (1997): "Does social capital have an economic payoff? Across-country investigation", The Quarterly Journal of Economics, 112(4), 1251-1288. DOI: 10.1162/003355300555475.

LIN, N. (1999): "Building a network theory of social capital", Connections, 22(1), 21-51.

LIN, N. (2005): "A network theory of social capital". In D. Castiglione, J. W. Van Deth \& G. Wolleb (Eds.), The Handbook of Social Capital, Oxford: Oxford University Press, 50-69.

MALECKI, E.J. (2012): "Regional social capital: why it matters", Regional Studies, 46(8), 1023-1039. DOI: 10.1080/00343404.2011.607806.

MALONEY, W. A. \& ROSSTEUTSCHER, S. (Eds.) (2007): Social capital and associations in European democracies: A comparative analysyis, Abingdon: Routledge. DOI: 10.4324/9780203969069.

MATTHEWS, R. \& FIDDLER, J. (2011): "Blood donation and community: exploring the influence of social capital", International Journal of Social Inquiry, 4(1), 45-63.

MCCAB, B.J. (2013): "Are homeowners better citizens? Homeownership and community participation in the United States", Social Forces, 91(3), 929-954. DOI: 10.1093/sf/sos185.

MCDONALD, S. \& MAIR, C.A. (2010): "Social capital across the life course: age and gendered patterns of network resources", Sociological Forum, 25(2), 335-359. DOI: 10.1111/j.15737861.2010.01179.x.

MELLSTRÖM, C. \& JOHANNESSON, M. (2008): "Crowding out in blood donation: was Titmuss right?", Journal Of the European Economic Association, 6(4), 845-863. DOI: 10.1162/jeea.2008.6.4.845.

MONTERO, J.R., ZMERLI, S. \& NEWTON, K. (2008): "Confianza social, confianza política y satisfacción con la democracia", Revista Española de Investigaciones Sociológicas, 122, 11-54. DOI: $10.2307 / 40184879$.

MONTESINOS SANTALUCÍA, V., PÉREZ GARCÍA, F., SERRANO MARTíNEZ, L. \& FERNÁNDEZ DE GUEVARA, J. (2005): La Medición del Capital Social: Una aproximación económica, Bilbao: Fundación BBVA. 
MONTOLIO, D. \& TUR-PRATS, A. (2018): "Long-lasting social capital and its impact on economic development: the legacy of the commons", IEB Working Paper, 2018/16. DOI: 10.2139/ssrn.3266640.

MORGAN, A. \& HAGLUND, B.J.A. (2009): "Social capital does matter for adolescent health: Evidence from the English HBSC study", Health Promotion International, 24(4), 363-372. DOI: 10.1093/ heapro/dap028.

NICHOLS, T.M. (1996): "Russian democracy and social capital", Social Science Information, 35(4), 629-642. DOI: 10.1177/053901896035004003.

PAARLBERG, L.E., HOYMAN, M. \& MCCALL, J. (2018): "Heterogeneity, income inequality, and social capital: a new perspective", Social Science Quarterly, 99(2), 699-710. DOI: 10.1111/ssqu.12454.

PEIRÓ PALOMINO, J. \& TORTOSA-AUSINA, E. (2012): "Social capital, investment and economic growth. Evidence for Spanish provinces", Fundación BBVA Working paper, 14. DOI: 10.2139/ ssrn.2222722.

PETRO, N.N. (2001): "Creating Social Capital in Russia: The Novgorod Model", World Development, 29(2), 229-244. DOI: 10.1016/s0305-750x(00)00101-7.

POEL, C.L., SEIFRIED, E. Van der \& SCHAASBERG, W.P. (2002): "Paying for blood donations: still a risk?" Vox Sanguinis, 83(4), 285-293. DOI: 10.1046/j.1423-0410.2002.00239.x.

PUTNAM, R.D. (1993): Making Democracy Work: Civic Traditions in Modern Italy, Princeton: Princeton University Press.

PUTNAM, R.D. (2000): Bowling alone: The collapse and revival of American community, New York: Simon and Schuster Paperbacks.

PUTNAM, R.D. (2007): "E Pluribus Unum: Diversity and Community in the Twenty-first Century (The 2006 Johan Skytte Prize Lecture)", Scandinavian Political Studies, 30(2), 137-174. DOI: 10.1111/j.1467-9477.2007.00176.x.

PUTNAM, R.D., LEONARDI, R., NANETTI, R.Y. \& PAVONCELLO, F. (1983): "Explaining institutional success: the case of Italian regional government", American Political Science Review, 77(1), 5574. DOI: $10.2307 / 1956011$.

RUPASINGHA, A., GOETZ, S.J. \& FRESHWATER, D. (2000): "Social capital and economic growth: a county-level analysis", Journal of Agricultural and Applied Economics, 32(3), 565-572. DOI: 10.1017/s1074070800020654.

RUPASINGHA, A., GOETZ, S.J. \& FRESHWATER, D. (2006): "The production of social capital in US counties", The Journal of Socio-Economics, 35(1), 83-101. DOI: 10.1016/j.socec.2005.11.001.

SOLOW, R.M. (2000): "Notes on social capital and economic performance". In P. Dasgupta \& I. Serageldin (Eds.), Social capital: A multifaceted perspective, Washington DC: The World Bank, 6-10. DOI: 10.1596/978-0-8213-5004-1. 
SUNDQUIST, K., HAMANO, T., LI, X., KAWAKAMI, N., SHIWAKU, K. \& SUNDQUIST, J. (2014): "Linking social capital and mortality in the elderly: A Swedish national cohort study", Experimental Gerontology, 55, 29-36. DOI: 10.1016/j.exger.2014.03.007.

TOLSMA, J., MEER, T. Van Der \& GESTHUIZEN, M. (2009): "The impact of neighbourhood and municipality characteristics on social cohesion in the Netherlands", Acta Politica, 44(3), 286-313. DOI: 10.1057/ap.2009.6.

UPHOFF, N. (2000): "Understanding social capital: learning from the analysis and experience of participation". In P. Dasgupta \& I. Serageldin (Eds.), Social capital: A multifaceted perspective, Washington DC: World Bank, 215-249. DOI: 10.1596/978-0-8213-5004-1.

\section{Anexo. Enlaces a los mapas interactivos en línea:}

Participación electoral municipal, en porcentaje:

https://www.instamaps.cat/visor.html?businessid=3378a614ad3c73be59e2f0426e5ac5f5\&3D=false

Participación electoral comarcal, en porcentaje:

https://www.instamaps.cat/visor.html?businessid=36c8dba40041f148dde605e889bcc316\&3D=false

Densidad asociativa municipal, en asociaciones por 1000 habitantes:

https://www.instamaps.cat/visor.html?businessid=1bfef5759b11cc1f3667e6bdf3eaa9c5\&3D=false

Densidad asociativa comarcal, en asociaciones por 1000 habitantes:

https://www.instamaps.cat/visor.html?businessid=5dc586a99d67695cb6e5139175ff9f11\&3D=false

Donaciones medias de sangre municipales, en donaciones por 1000 habitantes:

https://www.instamaps.cat/visor.html?businessid=f0a5ac7874f3e10911d6b1102050a054\&3D=false

Donaciones medias de sangre comarcales, en donaciones por 1000 habitantes:

https://www.instamaps.cat/visor.html?businessid=0943e3bf2215792bda64bb31b048db6b\&3D=false

Índice de capital social municipal:

https://www.instamaps.cat/visor.html?businessid=f26bcd07a2e090329952a85b63831348\&3D=false

Índice de capital social comarcal:

https://www.instamaps.cat/visor.html?businessid=84e7246862c05f48b26d32267dfa190b\&3D=false 